\title{
Peptide Metal-Organic Frameworks for enantioselective separation of chiral drugs
}

José Navarro-Sánchez ${ }^{\mathrm{a} \ddagger}$, Ana I. Argente-García ${ }^{\mathrm{b} \ddagger}$, Yolanda Moliner-Martínez $z^{\mathrm{b}}$, Daniel Roca-Sanjuán ${ }^{\mathrm{a}}$, Dmytro Antypov ${ }^{\mathrm{c}}$, Pilar CampínsFalcó, ${ }^{b}$ Matthew J. Rosseinsky ${ }^{c}$ and Carlos Martí-Gastaldo ${ }^{* a}$

${ }^{a}$ Universidad de Valencia (ICMol), Catedrático José Beltrán-2, 46980, Paterna (Spain)

${ }^{\text {b} M I N T O T A ~ r e s e a r c h ~ g r o u p . ~ D e p a r t a m e n t ~ d e ~ Q u i ́ m i c a ~ A n a l i ́ t i c a, ~ F a c u l t a t ~ d e ~ Q u i ́ m i c a, ~ U n i v e r s i t a t ~ d e ~ V a l e n c i a, ~ D o c t o r ~ M o l i n e r, ~ 50, ~} 46100$ Burjassot, (Spain).

'Department of Chemistry, University of Liverpool. Crown Street, Liverpool, L697ZD (UK)

E-mail: carlos.marti@uv.es

Metal-Organic Frameworks, enantioselective separation, chiral recognition, ephedrine, solid-phase extraction

\begin{abstract}
We report the ability of a chiral Cu(II) 3D MOF based on the tripeptide Gly-L-His-Gly (GHG) for the enantioselective separation of metamphetamine and ephedrine. Monte Carlo simulations suggest that chiral recognition is linked to preferential binding of one of the enantiomers as result of either stronger or additional H-bonds with the framework that lead to energetically more stable diastereomeric adducts. Solid phase extraction (SPE) of a racemic mixture by using $\mathrm{Cu}(\mathrm{GHG}$ ) as extractive phase permits isolating more than $50 \%$ of the $(+)$-ephedrine enantiomer as target compound in only four minutes. To the best of our knowledge, this represents the first example of a MOF capable of separating chiral polar drugs.
\end{abstract}

Metal-Organic Frameworks (MOFs) are crystalline, porous materials built from the interconnection of metal ions or clusters and organic linkers to produce porous architectures. Besides other applications like catalysis or sensing, their unlimited structur$\mathrm{al} /$ chemical flexibility enables rational tailoring of the chemical function and pore dimensions for more efficient and selective separation of gases, ${ }^{1}$ hydrocarbons ${ }^{2,3}$ or aromatic compounds. ${ }^{4}$ In this context, separation of chiral molecules with biological activity is also very relevant. A large number of drug compounds are often dosed as racemic mixtures. Enantiomers generally display different pharmacological and/or toxicological properties which can lead to a loss in activity or even to undesired side-reactions. Discovery of chiral adsorbents that permit rapid separation of individual enantiomers is of utmost importance to the early stages of drug research in the pharmaceutical industry. This type of separation relies on chiral recognition rather than shape/size selectivity. Hence, preferential interaction of one of the enantiomers with the homochiral adsorbent must lead to the formation of a transient diastereomeric adsorbate, whose free energy shall be sufficiently different for enantiomeric separation to take place.

Compared to classical adsorbents like zeolites or activated carbons, MOFs are arguably more promising candidates for chiral separation. They can be more easily engineered to deploy a periodic array of chiral channels that are accessible to guest sorption and can be modified in size, shape and chemical function to optimize mass transfer rates and chiral recognition. However, the application of MOFs in enantioselective separation remains still limited by small number of homochiral, microporous phases available. ${ }^{5,6}$ These are typically prepared either by using chiral templates that drive enantiomeric resolution, by post-synthetic linker exchange for partial grafting of chiral linkers in the metal struts at expense of accessible porosity or direct synthesis from enantiopure linkers. ${ }^{5}$ This last route is compatible with the use of naturally occurring chiral linkers like camphoric and tartaric acid, amino acids (aa's) or oligopeptides. Among these, peptides are well suited to producing functional, chiral MOFs whose robustness, chemical stability and porous response can be effectively modulated by suitable choice of aa's in the peptidic sequence. ${ }^{7-10}$ The broad choice of sidechain groups available from proteinogenic aa's can be also used to engineer the chiral pockets in their structure with specific functions. Due to their crystalline nature, peptide MOFs are also well-suited for theoretical modelling of the enantiomer interactions in a confined space, that is important to accelerate screening of materials already available and guide the design of more efficient systems.

Metamphetamine (MA) and ephedrine (EP) are chiral compounds of use as recreational drugs, but also as components in pharmaceutical preparations of therapeutic use as bronchodilators and respiratory stimulants.

Both compounds possess a

chiral center and exist as a pair of enantiomers which may differ in their pharma- Scheme 1. Structure of MA and EP. cological activity as well as in their metabolic and pharmacokinetic characteristics. Separation of MA or EP enantiomers is frequently required in clinical, forensic and toxicological laboratories. We report the ability of a chiral $\mathrm{Cu}$ (II) 3D MOF based on the tripeptide Gly-L-His-Gly (GHG) for their separation and provide clear insights on the experimental stereo selectivity by theoretical simulations, that reveal how intermolecular interactions between the drugs and the peptide backbone guide chiral recognition. We are confident our results might guide the development of efficient porous media for enantioselective applications.

$\mathrm{Cu}(\mathrm{GHG})$ was synthesized by slow diffusion of glycyl-Lhistidylglycine and $\mathrm{Cu}(\mathrm{II})$ acetate to produce prismatic, micrometric blue crystals by following a synthetic procedure recently reported by some of us. ${ }^{11}$ As shown in Figures SI1-4, phase purity was 
a

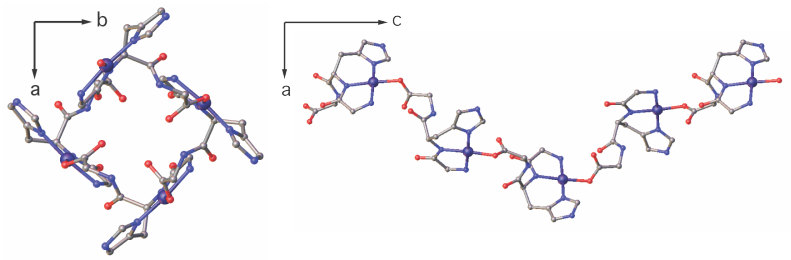

b
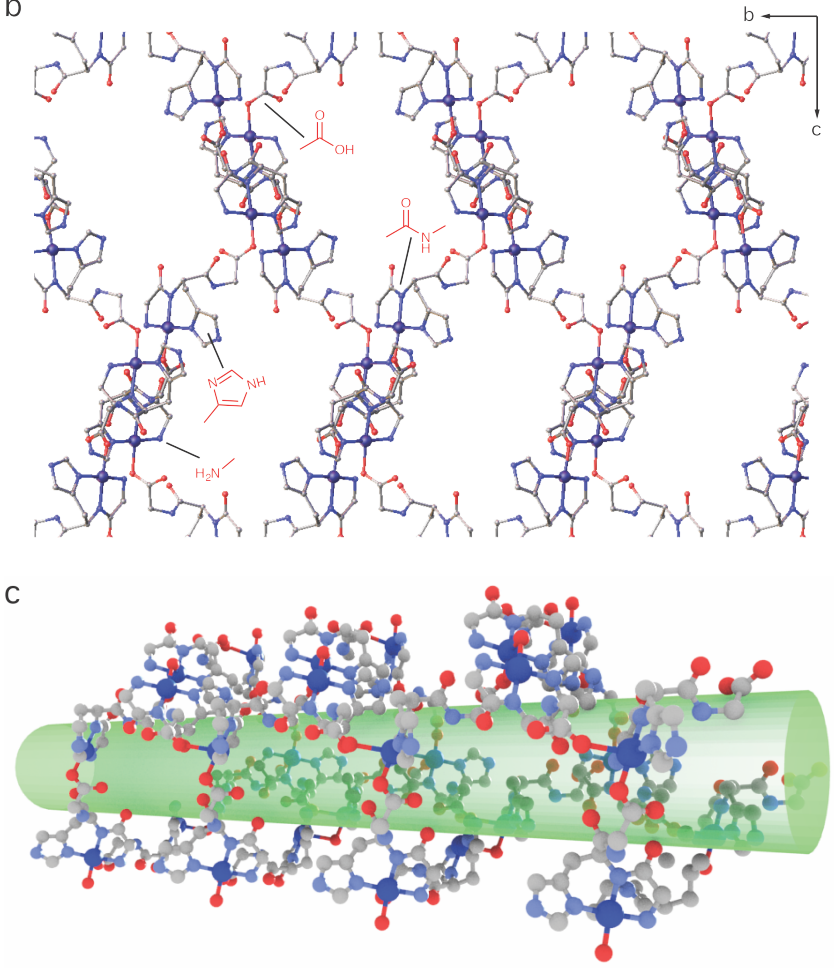

Figure 1. (a) Structure of helicoidal chains in Cu(GHG) MOF. (b) Functional groups in the peptidic backbone decorating the surface of the pores. (c) $1 \mathrm{D}$ channels in $\mathrm{Cu}(\mathrm{GHG})$ are surrounded by functional sites prone to establish supramolecular interactions, well fitted for chiral recognition and discrimination.

studied by CHN, FT-IR, Scanning Electron Microscopy (SEM), thermogravimetric analysis and powder X-ray diffraction (PXRD). Analysis of the PXRD collected at room temperature is consistent with the single-crystal data available, thus confirming the isolated solid is isostructural with the phase reported. ${ }^{11}$ Homochirality of the solid was also confirmed by solid-state circular dichroism (CD), that displays a positive Cotton effect in the UV-Vis spectrum of the MOF at 610 $\mathrm{cm}^{-1}$ linked to $\mathrm{Cu}(\mathrm{II}) \quad$ complexation with enantiopure glycil-Lhistidylglicine (Figure SI5).

$\mathrm{Cu}(\mathrm{GHG})$ crystallizes in the tetragonal, polar space group $P 4{ }_{1} 2{ }_{1} 2$ to produce an open 3D framework, built from the interconnection of 4-fold helicoidal $\mathrm{Cu}$-peptide-Cu chains by $\mu_{2}$-carboxylate bridges in $\mathrm{C}$ term Gly (Figure 1a). Porosity arises from the interconnection of $1 \mathrm{D}$ empty channels that account for a solvent accessible volume close to $60 \%$ of the total volume. Use of peptides as metal connectors enables to decorate the surface of the empty space with carboxylate, amide, amino and imidazole groups pointing inwards the channels (Figure 1b). This renders chemically versatile channels featuring a manifold of interaction sites for modulating framework/guest interactions (Figure 1c). Study of the porosity metrics with Zeo++ yields diameters of the largest included $\left(D_{i}\right)$ and free spheres $\left(D_{f}\right)$ above $2 \mathrm{~nm}$, big enough to accommodate small drugs like ephedrine or methamphetamine (Figure SI6). For these guests, recognition can potentially be triggered by preferential sorption in the chiral pockets of the MOF rather than interactions at the surface of the solid.

Monte Carlo (MC) simulations were used for a better understanding of the role played by the functional groups from the peptide in controlling preferential adsorption for more favorable binding sites. We simulated the adsorption of the two enantiomers $(+,-)$ of MA and EP (Figure 2). Structure of $\mathrm{Cu}(\mathrm{GHG}$ ) (CCDC 961607) was first prepared for the theoretical study by removal of solvent molecules occupying the pores. Most stable conformations of MA and EP enantiomers were explored by randomly changing the torsion angles. Next, MC computations were carried for each enantiomer to determine their most stable locations within the MOF upon sorption (See SI2 for details). Figure 2 summarizes the adsorption energies and preferential binding sites for the most representative host-guest adducts calculated. Adsorption of both MA enantiomers is preferentially directed by the formation of hydrogen bonds between the amine and the imidazole group in His sidechain. We have used discrete models of the MOF and quantum-chemistry calculations to confirm that this interaction is energetically most favorable, at least $4 \mathrm{kcal} . \mathrm{mol}^{-1}$ stronger, than those accessible by interaction with other functional groups in the pocket involving C-term and N-term Gly (Figure SI7, Table SI1). Regarding enantiomeric recognition, (+)-MA is accommodated closer to the pocket for a stronger interaction via imidazole $\mathrm{H}$-bond. This elongates from $1.87 \AA$ for (+)-MA to $2.03 \AA$ when interacting with (-)-MA, due to small changes in the adsorption configurations of the enantiomers (Figure 2). The higher binding energy observed for (+)-MA mainly arises from stronger van der Waals interactions

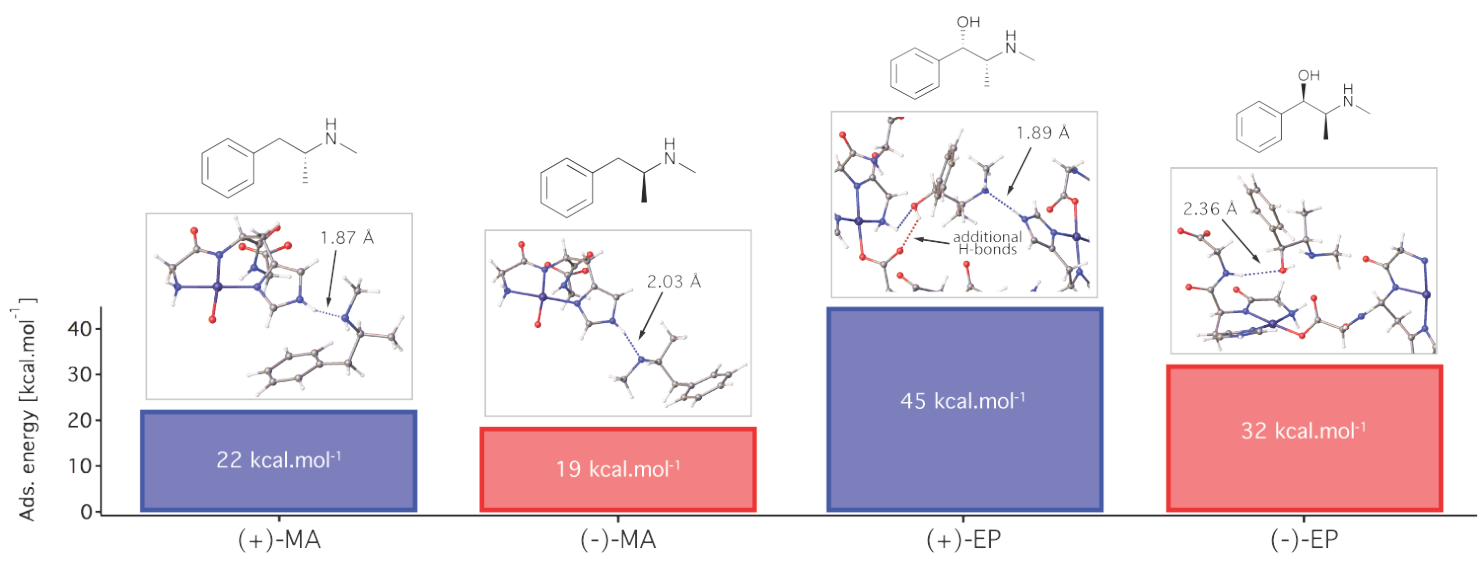

Figure 2 Representative MC binding geometries of (+,-)MA and EP enantiomers within the structure of $\mathrm{Cu}(\mathrm{GHG})$ and corresponding adsorption energies as absolute values calculated with respect to gas phase. Most relevant H-bonds in directing guest binding are annotated in each case. See Figure SI9 for an overall view of geometries and more favorable binding sites in the framework. Dotted lines represent H-bonds with N-H (blue) and O-H (red) donor groups. 
due to a better fit to the shape of the pocket. This agrees well with the energy variation for increasing $\mathrm{H}$-bond distances calculated by quantum-chemistry methods for short range imidazoledimethylamine interactions (Figure SI8). While the accuracy of the force-field model and the difficulty of replicating experimental solvation effects do not allow for quantitative prediction of chiral selectivities, our simulations suggest that more effective enantioselective recognition is expected for ephedrine as result of cooperative supramolecular interactions. As shown in Figure 2, adsorption of (-)-EP is solely directed by the formation of a weak elongated $\mathrm{H}$ bond $(2.36 \AA)$ with the amide bond in C-term Gly. In contrast, (+)-EP combines short His-EP H-bonds (1.89 ̊), equivalent to that with (+)-MA, with auxiliary bonds with the carboxylate and amino groups (2.09 and $2.40 \AA$ ) in the C-term and N-term Gly amino acids of $\mathrm{Cu}(\mathrm{GHG})$ for more favorable adsorption. It is the strength and number of H-bonds, EP has two hydrogen bonding groups capable of interacting synergically with the environment of the peptide MOF, that translates into a bigger difference in adsorption energies close to $13 \mathrm{kcal}_{\mathrm{mol}}{ }^{-1}$.

MC simulations suggest that $\mathrm{Cu}(\mathrm{GHG})$ might display stereo selective, in particular for EP enantiomers, associated with the control of non-covalent interactions over their binding geometries. This promising result prompted us to attempt actual separation experiments from solutions of the racemates. One of the key limitations of flexible MOFs is their poor mechanical robustness that results in collapse of the structure upon solvent removal. This is also the case for $\mathrm{Cu}-\mathrm{GHG}$ which displays sponge-like behavior with a crystallineto-amorphous transformation after activation that can be reverted to the original state in presence of polar solvents. ${ }^{11}$ Though this is a key limitation for gas storage, which requires the porous material to be activated prior to gas sorption, chiral recognition is a dynamic process and prior filling of the pores is not imperative since the solvent molecules occupying the pores can be progressively exchanged with the chiral guests in solution. Hence, all separation experiments were carried out by using non-evacuated, assynthesized materials in order to preserve their structural integrity. First, we studied the ability of $\mathrm{Cu}(\mathrm{GHG})$ to recognize selectively enantiomers from solution by soaking $10 \mathrm{mg}$ of fresh solid in racemic mixtures of MA and EP (40 $\mu \mathrm{g} / \mathrm{mL}$ in ethanol:water 75:25). Enantioselective recognition was evaluated chromatographically as a function of contact time (See SI3 for experiment conditions and methodology). As shown in Figure 3, adsorption of (-)-MA and ()-EP can be considered negligible whereas $30 \pm 3 \%$ of $(+)-\mathrm{MA}$ and $37 \pm 3 \%(+)$-EP are preferentially adsorbed after 4 and 2 hours, respectively. We attribute the long recognition times to the highly polar mixture of solvents used. This will stabilize the chiral drugs in solution preventing fast diffusion into the porous chiral medium for a slow recognition process with long equilibration times. This experiment agrees well with the MC simulations that support preferential interaction of $(+)$-enantiomers in the chiral pocket linked to specific conformations that enable $\mathrm{H}$-bond formation with the histidine sidechain. As described above, theoretical predictions are also consistent with more favorable separation of EP enantiomers over MA's based on the energy differences for enantiomer interaction with $\mathrm{Cu}(\mathrm{GHG})$ that are higher for the first. We also performed a control experiment by using a ground mixture of GHG and $\mathrm{Cu}(\mathrm{II})$ acetate in the same proportion present in the MOF. Individual components do not show measurable chiral recognition (Figure SI11), suggesting that the periodic distribution of chiral channels in the MOF scaffold is key for enantiomeric separation. In order to confirm the stability of the solid in the experiment conditions we collected UV-Vis spectra of dispersions of $\mathrm{Cu}(\mathrm{GHG})$ at

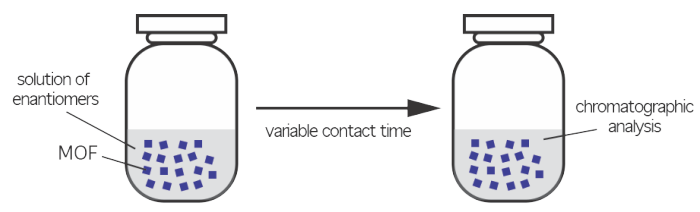

a

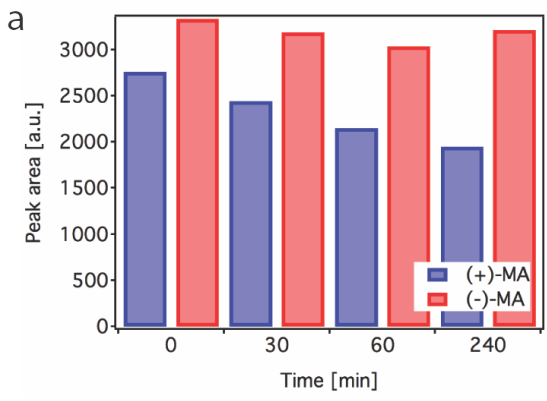

b

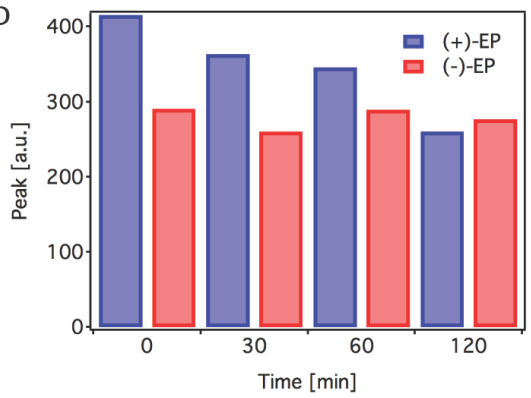

Figure 3. Evolution of the enantioselective recognition of $\mathrm{Cu}(\mathrm{GHG})$ for chiral drugs in ethanol:water 75:25 solution with contact time for: (a) MA and (b) EP. Chiral adsorption was evaluated from chromatographic analysis of the supernatant solution. See Fig.SI10 for more details.

variable time. As shown in Figure SI15, spectra remain constant and there is no signature of $\mathrm{Cu}$ (II) leaching that might account for partial dissolution. As for the MOF structure, PXRD of the solid after the experiment confirms the structure remains intact (Figure SI16a) with only minor variations in the relative intensities of some Bragg's reflections ascribed to preferential orientation of the crystals or changes in the nature of the species occupying the pores.

Recent reports highlight the potential of chiral MOFs for the development of CSPs in the form of packed columns for highperformance liquid chromatography (HPLC) enantioselective separation. ${ }^{12-17}$ However, the limited number of tests available suggest that the main limitations of MOFs as stationary phases are their poor stability and particle heterogeneity. This limits the range of mobile phases at choice, in particular for polar analytes, and makes it difficult to achieve efficient packing of the particles in the column that relies on narrow distributions in size and shape for reproducible sorption/desorption kinetics. Lack of control over these variables can result in high retention times, low chromatographic performance and energy consuming separations. MOFs might be instead better fitted for Solid Phase Extraction (SPE) separation technologies. In contrast to CSP, SPE enables isolation of the enantiomers rather than only providing a measure of the enantiomeric purity of a mixture. SPE can allow for more efficient chromatographic analysis by removal of interferences, increase of trace concentration or sample simplification but use of chiral MOFs in this context remains still unexplored. To illustrate this concept, we packed $50 \mathrm{mg}$ of $\mathrm{Cu}(\mathrm{GHG})$ in a polypropylene SPE cartridge and eluded a racemic mixture of $( \pm)$-EP in hexane:ethanol $(75: 25)$ at $0.25 \mathrm{~mL} \mathrm{~min}^{-1}$ followed by HPLC analysis of the resulting extract (Figure 4a). Experimental setup is shown in Figure SI12. We opted for EP rather than MA based on our prelim- 


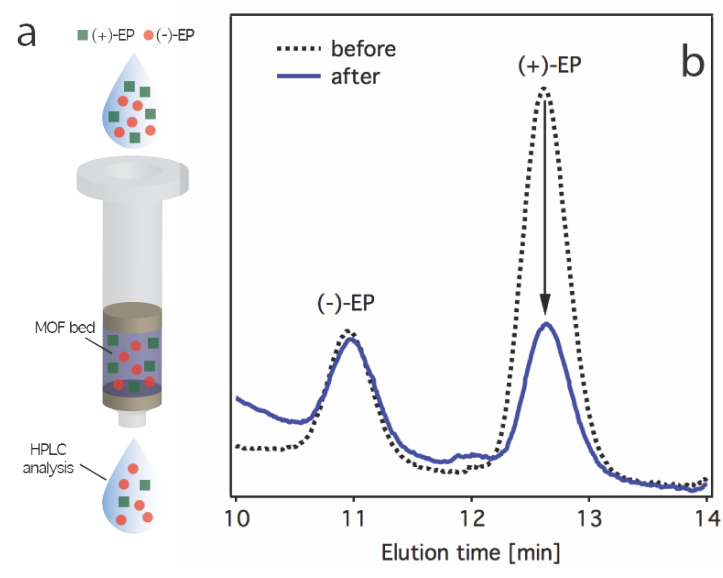

Figure 4. (a) SPE separation of EP in hexane:EtOH 75:25 by using $\mathrm{Cu}(\mathrm{GHG})$ as chiral bed. (b) HPLC chromatograms of EP racemate before (dashed line) and after (solid line) passing through the MOF bed. See SI 4 for experimental details.

inary results that suggest faster and more efficient recognition of the first. As shown in Figure $4 b$, comparison of the chromatograms of the sample before and after SPE/Cu(GHG) separation confirm the ability of the MOF to trap preferentially (+)-EP, in line with our theoretical predictions. While the peak area contribution for ()-EP remains constant, there is a significant decrease for the (+)form. Quantitatively, the chiral solid permits separating $54 \pm 2 \%$ of (+)-EP from equimolar mixtures of the enantiomers in 4 minutes with a satisfactory precision $(\mathrm{RSD}=4 \%)$ for the different cartridges tested (Table SI3). HPLC analysis of the solution desorbed from the MOF by elution with fresh solvent confirms that only (+)-EP enantiomers are retained in the separation, confirming enantioselectivity (Figure SI13). Compared to SPE, direct soaking of the crystals in a racemic mixture of $( \pm)$-EP in hexane:EtOH 75:25 also leads to enantioselective separation of $44 \%$ of $(+)$-EP in the same time (Figure SI14). MOF cartridges can be used at least for two runs without significant loss of enantioselectivity. PXRD (Figure SI16b-d) and SEM (Figure SI17) studies of the crystals in the MOF bed after separation experiments confirm that both, structure and morphology of the solid, remain unchanged. Hence, we ascribe loss of activity to an inefficient packing of the solid rather than to chemical degradation. Our results suggest that $\mathrm{Cu}(\mathrm{GHG})$ offers high performance in terms of stereo selective recognition and time efficiency, for a high-speed chiral separation medium.

Peptide MOFs are particularly well-suited for enantioselective recognition. The chiral pockets in their structure can be manipulated for maximizing host-guest intermolecular interactions for a specific enantiomer simply by suitable choice of the peptide sequence. $\mathrm{Cu}(\mathrm{GHG})$ is capable of separating more than $50 \%$ of $(+)$ ephedrine from a racemic mixture in only four minutes when used as a chiral SPE cartridge. This is a promising result regarding the limited availability of SPE cartridges for chiral separation in the market. According to our theoretical simulations, stereo selectivity is driven by preferential non-covalent interactions with specific amino acids, particularly His, in the MOF backbone. This combination of theory and experiment holds great potential and might help addressing the challenge of chiral recognition by producing more complex chemical environments in which supramolecular interactions can be finely tuned for maximizing enantiomeric resolution for particular drugs.

\section{ASSOCIATED CONTENT}

\section{Supporting Information}

The Supporting Information is available free of charge on the ACS Publications website.

Synthetic and experimental details. Physical characterization and supporting tables and figures (PDF)

\section{AUTHOR INFORMATION}

\section{Corresponding Author}

*carlos.marti@uv.es

\section{Author Contributions \\ J.N and A.I.A.-G. contributed equally.}

\section{ACKNOWLEDGMENT}

This work was supported by the EU (ERC Stg Chem-fs-MOF 714122), Spanish MINECO (Project CTQ-2014-53916-P and Unit of Excellence María de Maeztu MDM-2015-0538) and the Generalitat Valenciana (Prometeo 2016/109 and GV/2016/137). C.M.-G. and A.I.A.-G. thank the Spanish MINECO for a Ramón y Cajal Fellowship and FPU Scholarship, respectively.

\section{REFERENCES}

(1) Nugent, P.; Giannopoulou, E. G.; Burd, S. D.; Elemento, O.; Forrest, K.; Pham, T.; Ma, S.; Space, B.; Wojtas, L.; Eddaoudi, M.; Zaworotko, M. J. Nature 2013, 495, 80.

)
Hartlieb, K. J.; Holcroft, J. M.; Moghadam, P. Z.; Vermeulen, N. A.; Algaradah, M. M.; Nassar, M. S.; Botros, Y. Y.; Snurr, R. Q.; Stoddart, J. F. J. Am. Chem. Soc. 2016, 138, 2292. 
(13) Hailili, R.; Wang, L.; Qv, J.; Yao, R.; Zhang, X.-M.; Liu, H. Inorg. Chem. 2015, 54, 3713.

(14) Peng, Y.; Gong, T.; Zhang, K.; Lin, X.; Liu, Y.; Jiang, J.; Cui, Y. Nature Communications 2014, 5, 4406.

(15) Zhang, M.; Pu, Z.-J.; Chen, X.-L.; Gong, X.-L.; Zhu, A.X.; Yuan, L.-M. Chem. Commun. 2013, 49, 5201.

(16) Das, M. C.; Guo, Q.; He, Y.; Kim, J.; Zhao, C.-G.; Hong,
K.; Xiang, S.; Zhang, Z.; Thomas, K. M.; Krishna, R.; Chen, B. J. Am. Chem. Soc. 2012, 134, 8703.

Padmanaban, M.; Müller, P.; Lieder, C.; Gedrich, K.; Grünker, R.; Bon, V.; Senkovska, I.; Baumgärtner, S.; Opelt, S.; Paasch, S.; Brunner, E.; Glorius, F.; Klemm, E.; Kaskel, S. Chem. Commun. 2011, 47, 12089.

Table of Contents artwork

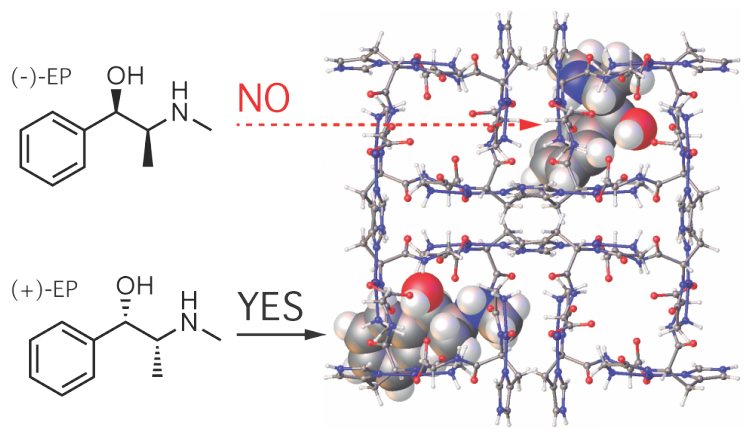

